Article

\title{
Anti-Inflammatory Effects of Low-Dose Glucocorticoids Compensate for Their Detrimental Effects on Bone Mineral Density in Patients with Rheumatoid Arthritis
}

\author{
Ji-Won Kim ${ }^{1}$, Ju-Yang Jung ${ }^{1}$, Hyoun-Ah $\operatorname{Kim}^{1}{ }^{1}$ and Chang-Hee Suh ${ }^{1,2, *(\mathbb{D})}$ \\ 1 Department of Rheumatology, Ajou University School of Medicine, Suwon 16499, Korea; \\ jwk722@naver.com (J.-W.K.); serinne20@hanmail.net (J.-Y.J.); nakhada@naver.com (H.-A.K.) \\ 2 Department of Molecular Science and Technology, Ajou University, Suwon 16499, Korea \\ * Correspondence: chsuh@ajou.ac.kr; Tel.: +82-31-219-5118; Fax: +82-31-219-5157
}

check for updates

Citation: Kim, J.-W.; Jung, J.-Y.;

Kim, H.-A.; Suh, C.-H.

Anti-Inflammatory Effects of

Low-Dose Glucocorticoids

Compensate for Their Detrimental Effects on Bone Mineral Density in Patients with Rheumatoid Arthritis. J. Clin. Med. 2021, 10, 2944. https:// doi.org/10.3390/jcm10132944

Academic Editor: Emmanuel Andrès

Received: 18 June 2021

Accepted: 29 June 2021

Published: 30 June 2021

Publisher's Note: MDPI stays neutral with regard to jurisdictional claims in published maps and institutional affiliations.

Copyright: (c) 2021 by the authors. Licensee MDPI, Basel, Switzerland. This article is an open access article distributed under the terms and conditions of the Creative Commons Attribution (CC BY) license (https:/ / creativecommons.org/licenses/by/ $4.0 /)$.

\begin{abstract}
Objectives: This study aimed to provide reliable information on the impact of low-dose glucocorticoids (GCs) on the bone mineral density (BMD) of patients with rheumatoid arthritis (RA). Methods: This retrospective study enrolled 933 patients with RA who continued the consumption of GCs (GC group) and 100 patients who had discontinued consumption for $>1$ year (no-GC group). The BMD values were measured at baseline and follow-up, and the annual rate of change in BMD between the groups was compared using dual-energy X-ray absorptiometry. We used multiple linear regression analysis to identify the factors associated with changes in BMD. Results: The demographic characteristics and use of medical treatments affecting bone metabolism were similar between the two groups. Furthermore, there were no significant differences in the annual rate of changes in BMD and incidence of newly developed osteoporosis and incidental fractures between the two groups. Multiple linear regression analysis revealed that the disease activity score for 28 joints with erythrocyte sedimentation rate was the only factor affecting the annual rate of changes in BMD, and it was inversely proportional to changes in BMD. Conclusion: The benefits of GC therapy in attenuating inflammation compensate for the risk of osteoporosis if adequate measures to prevent bone loss are implemented in patients with RA.
\end{abstract}

Keywords: rheumatoid arthritis; glucocorticoids; osteoporosis; disease activity

\section{Introduction}

Rheumatoid arthritis (RA) is a systemic autoimmune disease characterized by chronic, symmetrical, and progressive inflammatory polyarthritis [1]. Under pathological conditions of RA, the balance between bone resorption and formation is disrupted by the expression of pro-inflammatory cytokines that promote osteoclast differentiation and suppress the osteogenic activity of the osteoblasts [2]. Moreover, uncontrolled inflammation is transformed into hyperplastic invasive tissue, which destroys the cartilage and bone, leading to local and generalized bone loss [3]. This mechanism causes osteoporosis, a major complication of RA, which is a risk factor for fractures, impairs functional ability, deteriorates the quality of life, and further contributes to increased mortality [4].

Over the past few decades, glucocorticoids (GCs) have continued to play an important role in the treatment of various inflammatory diseases. GCs are widely recognized for their role as combination drugs in RA treatment and are considered highly effective in reducing the signs and symptoms of the disease and maintaining low disease activity [5]. However, GCs significantly contribute to the development of osteoporosis in patients with RA by decreasing the number of osteoblasts and promoting apoptosis of osteoblasts and osteocytes [6]. In most previous studies, GC-treated patients with RA had lower bone density and higher incidence rates of osteoporosis and fracture than the general population or patients with RA not treated with GCs [7]. However, high disease activity is another 
important risk factor that increases the incidence of osteoporosis, similar to GC therapy. Hence, it is often necessary to maintain low-dose GC therapy along with disease-modifying anti-rheumatic drugs (DMARDs) to achieve high and persistent remission rates [8].

Nevertheless, the prevailing opinion is that it is beneficial to discontinue GCs as soon as possible rather than maintain the remission state by administering low-dose GCs $[9,10]$. To provide reliable information on the effect of low-dose GCs on bone mineral density (BMD), we investigated the changes in BMD and disease activity, new-onset osteoporosis rates, and incidence of fractures during the maintenance of low-dose GC therapy in patients with RA. Furthermore, we analyzed other risk factors that could accelerate the reduction in BMD.

\section{Materials and Methods}

\subsection{Study Design and Population}

In this retrospective study, we identified 1480 patients with a record of low-dose GC use ( $<7.5 \mathrm{mg} /$ day of prednisone or equivalent) among patients with RA from the Ajou University Hospital medical records database [11]. We excluded 346 patients who did not meet the inclusion criteria or did not have sufficient data due to irregular visits. We reviewed data on 1234 eligible patients who visited Ajou University Hospital between January 1999 and June 2020. Finally, we included 933 patients, excluding those diagnosed with other rheumatic diseases or those receiving GCs for other causes. Figure 1 shows the flowchart of this study. The study group included 833 patients aged $>18$ years who fulfilled the 1987 American College of Rheumatology (ACR) or 2010 ACR/European League Against Rheumatism classification criteria for RA diagnosis and continued to take GCs during the study period. The control group included patients diagnosed with RA who had not taken GCs for $>1$ year prior to the baseline BMD test. This study was approved by the Institutional Review Board of Ajou University Hospital (AJIRB-MED-MDB-21-109).

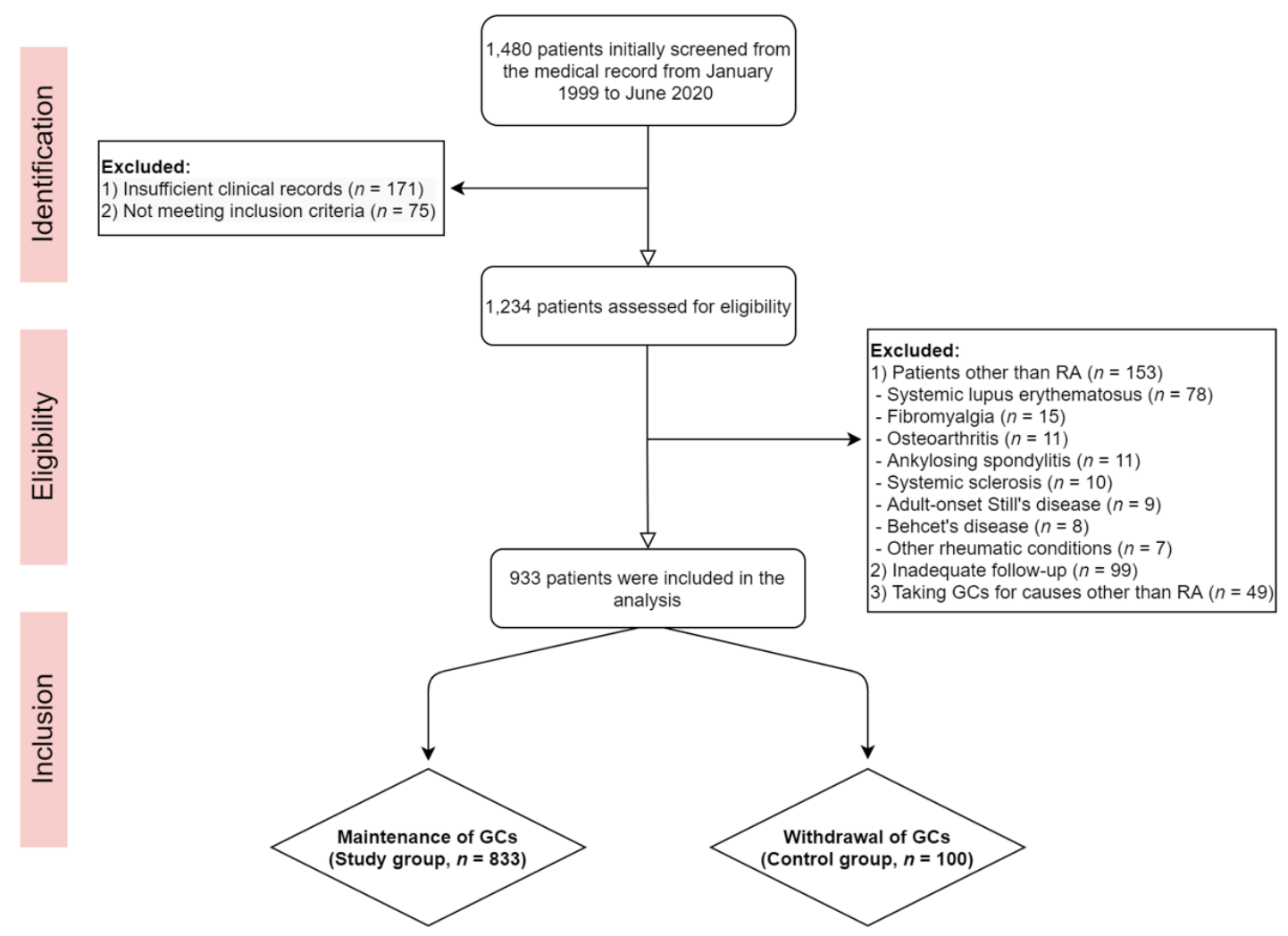

Figure 1. Flowchart of this retrospective study. RA: Rheumatoid arthritis, GCs: Glucocorticoids. 


\subsection{Clinical Assessments}

All participants underwent structured interviews, physical examinations, laboratory tests, medical record reviews, and radiologic tests. Demographic data included information on age, sex, body mass index (BMI), menopausal status, alcohol consumption, smoking habits, and concomitant diseases. Data on laboratory findings such as those for rheumatoid factor (RF), anti-citrullinated protein antibody, erythrocyte sedimentation rate (ESR), Creactive protein (CRP), and biochemistry parameters of the bone were collected. The medical record reviews helped to identify the duration of the disease, clinical characteristics (tender/swollen joint count and patient global assessment), and medications during followup, and the disease activity score of 28 joints (DAS28). The details of the assessment and treatment of the disease were documented by the rheumatologists. All radiographs were assessed by a radiologist, and bone erosion was defined as the presence of erosion in one or more of the proximal interphalangeal joints, metacarpophalangeal joints, wrists, and metatarsophalangeal joints on radiographs of the hand and foot. The imaging file provided to the radiologists did not include clinical information; therefore, the reading was performed in a clinically blind state.

\subsection{BMD and Fracture Measurements}

The BMD of the lumbar spine (L1-L4) and proximal femur (femoral neck and total hip) was measured at study enrollment and follow-up by dual energy X-ray absorptiometry (GE Lunar, Madison, WI, USA). All the scanners were operated according to the manufacturer's standard scanning software and positioning protocols. The absolute value of the BMD results is presented in grams per square centimeter $\left(\mathrm{g} / \mathrm{cm}^{2}\right)$ and additionally expressed as the T-score (compared to a normal young adult reference population) or Z-score (agematched controls) according to the World Health Organization (WHO) criteria. According to the WHO criteria, BMD is classified as normal, osteopenia, or osteoporosis based on a T-score (or Z-score) of -1 or above, between -1 and -2.5 (or -2 ), and below -2.5 (or -2 ), respectively [12]. BMD loss per year and the annual rate of change in BMD were calculated as absolute values.

A prior history of fractures (vertebral and non-vertebral) was assessed by radiologic evaluation and using patient self-reporting questionnaires. If the plain radiograph appeared normal at baseline but showed a fracture at follow-up, then it was considered an incidental fracture. Patients with fractures not related to osteoporosis or pathological fractures due to malignancy were excluded from the analysis.

\subsection{Statistical Analysis}

Data are presented as mean \pm standard deviation. The Wilcoxon rank test, the chisquare test, or Fisher's exact test was used to determine statistically significant differences in the variables between the GC and no-GC groups. Continuous variables were analyzed using the Wilcoxon rank test, and categorical variables were analyzed using the chi-square test or Fisher's exact test. Multiple linear regression analysis was used to investigate the factors affecting the annual rate of change in BMD. All statistical analyses were performed using R software, version 3.6.1 (R studio, Boston, MA, USA). For all analyses, $p<0.05$ was considered statistically significant.

\section{Results}

\subsection{Patient Characteristics}

The demographic and clinical characteristics of the patients at baseline are summarized in Table 1. There were 833 (89\%) patients who consumed low-dose GCs (GC group) and $100(11 \%)$ patients who had discontinued GCs for $>1$ year before the baseline BMD test (no-GC group). There was no significant difference between the two groups in terms of age, sex, menopausal status, BMI, smoking habits, alcohol consumption, or disease duration. However, compared to the no-GC group, the GC group had higher RF positivity, more pronounced elevation of DAS28-ESR, and the presence of bone erosion. The baseline bone 
biochemistry results did not deviate from the normal range and were similar between the two groups. There was a significant difference in the mean cumulative GC dose before BMD measurements between the groups, and the average doses were $7.68 \pm 6.58 \mathrm{~g}$ and $5.99 \pm 5.29 \mathrm{~g}$ of prednisone or an equivalent in the GC group and no-GC group, respectively. The mean daily GC dose before BMD measurements did not differ statistically between the groups $(4.11 \pm 2.6 \mathrm{mg} /$ day vs. $3.61 \pm 4.9 \mathrm{mg} /$ day of prednisone or an equivalent; $p=0.716$ ), and the mean GC usage during the follow-up period in the GC group was $2.23 \mathrm{mg} /$ day of prednisone or an equivalent. Methotrexate (MTX) and biological agents were used marginally more frequently in the GC group than in the no-GC group. The use of DMARDs other than MTX and non-steroidal anti-inflammatory drugs was similar between the groups.

Table 1. Baseline demographic and clinical characteristics of patients with RA.

\begin{tabular}{|c|c|c|c|}
\hline Variable & GC $(N=833)$ & No-GC $(\mathrm{N}=100)$ & $p$ Value \\
\hline Age, mean (years) & $63.7 \pm 9.8$ & $61.8 \pm 9$ & 0.062 \\
\hline \multicolumn{3}{|l|}{ Sex } & \multirow{2}{*}{0.15} \\
\hline Women, N. (\%) & $766(92)$ & $96(96)$ & \\
\hline Menopause, N. (\%) & $694(90.7)$ & $85(88.5)$ & 0.493 \\
\hline BMI, mean & $23.3 \pm 3.72$ & $23.8 \pm 3.33$ & 0.208 \\
\hline Smoking, N. (\%) & $48(5.8)$ & $9(9)$ & 0.203 \\
\hline Alcohol, N. (\%) & $91(10.9)$ & $11(11)$ & 0.985 \\
\hline RF positivity, N. (\%) & $563(67.3)$ & $51(51)$ & $0.001 *$ \\
\hline Anti-CCP Ab positivity, N. (\%) & $300(36)$ & $38(38)$ & 0.181 \\
\hline Disease duration (months) & $110.7 \pm 77.6$ & $100.2 \pm 58.8$ & 0.22 \\
\hline DAS28-ESR & $3.02 \pm 1.5$ & $2.56 \pm 0.9$ & $0.012 *$ \\
\hline DAS28-CRP & $2.19 \pm 0.98$ & $1.86 \pm 0.65$ & 0.062 \\
\hline Bone erosion, N. $(\%)$ & $329(39.4)$ & $21(21)$ & $<0.001$ * \\
\hline \multicolumn{4}{|c|}{ Bone biochemistry } \\
\hline Serum $25(\mathrm{OH}) \mathrm{D}(\mathrm{ng} / \mathrm{ml})$ (normal range 30.0-100.0) & $27.2 \pm 20.7$ & $29.3 \pm 11.3$ & 0.415 \\
\hline Serum calcium, (mg/dL) (normal range 8.6-10.2) & $9.52 \pm 4.28$ & $9.43 \pm 0.32$ & 0.834 \\
\hline Serum phosphate, $(\mathrm{mg} / \mathrm{dL})$ (normal range $2.5-4.5)$ & $3.52 \pm 0.56$ & $3.64 \pm 0.46$ & 0.054 \\
\hline Serum creatinine, $(\mathrm{mg} / \mathrm{dL})$ (normal range $0.5-0.9)$ & $0.78 \pm 0.39$ & $0.73 \pm 0.13$ & 0.085 \\
\hline Serum alkaline phosphatase, (U/L) (normal range 35-104) & $66.6 \pm 22.8$ & $66.4 \pm 19.8$ & 0.93 \\
\hline \multicolumn{4}{|l|}{ Medication } \\
\hline \multicolumn{4}{|l|}{ Glucocorticoids } \\
\hline Cumulative dose before BMD measurements (g) & $7.68 \pm 6.58$ & $5.99 \pm 5.29$ & 0.004 * \\
\hline Mean dose before BMD measurements (mg/day) & $4.11 \pm 2.6$ & $3.61 \pm 4.9$ & 0.716 \\
\hline Mean dose between BMD measurements (mg/day) & $2.23 \pm 1.69$ & NA & NA \\
\hline NSAIDs, N. (\%) & $560(67.2)$ & $61(61)$ & 0.219 \\
\hline Methotrexate, N. (\%) & $549(66)$ & $39(39)$ & $<0.001 *$ \\
\hline Hydroxychloroquine, N. (\%) & $500(60)$ & $70(70)$ & 0.053 \\
\hline Sulfasalazine, N. (\%) & $53(6.4)$ & $9(9)$ & 0.317 \\
\hline Tacrolimus/cyclosporin, N. (\%) & $174(20.9)$ & $13(13)$ & 0.063 \\
\hline Leflunomide, N. (\%) & $144(17.2)$ & $11(11)$ & 0.119 \\
\hline Anti-TNF agents, N. (\%) & $38(4.6)$ & $1(1)$ & $0.048^{*}$ \\
\hline Adalimumab, N. (\%) & $10(1.2)$ & $0(0)$ & 0.612 \\
\hline Etanercept, N. (\%) & $9(1.1)$ & $1(1)$ & $>0.999$ \\
\hline Golimumab, N. (\%) & $8(1)$ & $0(0)$ & $>0.999$ \\
\hline Infliximab, N. (\%) & $11(1.3)$ & $0(0)$ & 0.619 \\
\hline
\end{tabular}


Table 1. Cont.

\begin{tabular}{|c|c|c|c|}
\hline Variable & $\mathrm{GC}(\mathrm{N}=833)$ & No-GC $(N=100)$ & $p$ Value \\
\hline Abatacept, N. (\%) & $4(0.5)$ & $0(0)$ & 0.34 \\
\hline Tocilizumab, N. (\%) & $17(2.0)$ & $0(0)$ & 0.242 \\
\hline Rituximab, N. (\%) & $8(1)$ & $0(0)$ & $>0.999$ \\
\hline JAK inhibitors, N. (\%) & $11(1.3)$ & $0(0)$ & 0.619 \\
\hline
\end{tabular}

RA: rheumatoid arthritis, GC: glucocorticoid, BMI: body mass index, RF: rheumatoid factor, Anti-CCP Ab: anti-citrullinated protein antibody, DAS: disease activity score, ESR: erythrocyte sedimentation rate, CRP: C-reactive protein, 25(OH) D: 25-hydroxyvitamin D, BMD: bone mineral density, NSAIDs: non-steroidal anti-inflammatory drugs, TNF: tumor necrosis factor, JAK: Janus kinase, NA: not applicable. $* p<0.05$.

Table 2 presents the results of BMD measurements at baseline and osteoporosis treatments. The measurement results of the subregions are presented in grams per square centimeter $\left(\mathrm{g} / \mathrm{cm}^{2}\right)$ and as the T- or Z-score. The BMD of the lumbar spine was comparable between the groups, but that of the femoral neck and total hip was significantly lower in the GC group than in the no-GC group. There was no significant difference in the number of patients with osteoporosis according to the WHO criteria between the groups $(407(48.9 \%)$ vs. $39(39 \%) ; p=0.061)$. The proportion of patients treated with medications affecting bone metabolism, including vitamin D, calcium, and proton pump inhibitors, was similar in both groups. Bisphosphonates were the most commonly taken drugs for the treatment for osteoporosis in both groups, followed by selective estrogen receptor modulators, denosumab, and teriparatide.

Table 2. Baseline bone mineral density and osteoporosis treatments of patients with RA.

\begin{tabular}{|c|c|c|c|}
\hline Variable & $\mathrm{GC}(\mathrm{N}=833)$ & No-GC (N = 100) & $p$ Value \\
\hline \multicolumn{4}{|l|}{ Baseline BMD $\left(\mathrm{g} / \mathrm{cm}^{2}\right)$} \\
\hline Lumbar spine & $0.94 \pm 0.16$ & $0.95 \pm 0.16$ & 0.813 \\
\hline Femoral neck & $0.75 \pm 0.12$ & $0.78 \pm 0.11$ & $0.022 *$ \\
\hline Total hip & $0.8 \pm 0.14$ & $0.85 \pm 0.12$ & 0.001 * \\
\hline \multicolumn{4}{|l|}{ Baseline BMD, T-score } \\
\hline Lumbar spine & $-1.83 \pm 1.29$ & $-1.64 \pm 1.4$ & 0.55 \\
\hline Femoral neck & $-1.55 \pm 0.98$ & $-1.33 \pm 0.88$ & 0.033 * \\
\hline Total hip & $-1.38 \pm 1.13$ & $-0.99 \pm 1.01$ & $0.002 *$ \\
\hline \multicolumn{4}{|l|}{$\begin{array}{l}\text { WHO classification using BMD } \\
\text { measurements }\end{array}$} \\
\hline Normal, N. (\%) & $107(12.8)$ & $13(13)$ & 0.965 \\
\hline Osteopenia, N. (\%) & $319(38.3)$ & $48(48)$ & 0.06 \\
\hline Osteoporosis, N. (\%) & $407(48.9)$ & $39(39)$ & 0.062 \\
\hline Interval between BMDs (months) & $19.5 \pm 12.1$ & $19.9 \pm 12$ & 0.538 \\
\hline Calcium intake, N. (\%) & $317(38.1)$ & $47(47)$ & 0.103 \\
\hline Vitamin D intake, N. (\%) & $724(86.9)$ & $94(94)$ & 0.053 \\
\hline Proton pump inhibitor, N. (\%) & $170(20.4)$ & $16(16)$ & 0.354 \\
\hline Treatment for osteoporosis, N. (\%) & $411(49.3)$ & $40(40)$ & 0.077 \\
\hline Bisphosphonate, N. (\%) & $323(78.6)$ & $28(70)$ & 0.036 * \\
\hline SERM, N. $(\%)$ & $70(17)$ & $11(27.5)$ & 0.384 \\
\hline Denosumab, N. (\%) & $26(6.3)$ & $1(3.6)$ & 0.348 \\
\hline Teriparatide, N. (\%) & $2(0.5)$ & $0(0)$ & $>0.999$ \\
\hline
\end{tabular}

RA: rheumatoid arthritis, GC: glucocorticoid, BMD: bone densitometry, WHO: World Health Organization, SERM: selective estrogen receptor modulator. ${ }^{*} p<0.05$. 


\subsection{Effects of Corticosteroid Exposure on BMD and Osteoporotic Fractures}

The absolute differences between the baseline and follow-up BMD values and the annual rate of change in BMD at all measurement sites are presented in Table 3. In both groups, the BMD values of the lumbar spine increased, whereas those of the femoral neck and total hip decreased marginally, but without statistically significant differences between the groups. In the GC and no-GC groups, the annual rates of change in the BMD of the lumbar spine increased by $1.7 \%$ and $1.3 \%$, respectively, whereas those of the femoral neck decreased by $0.33 \%$ and $0.53 \%$, respectively, and those of the total hip decreased by $0.38 \%$ and $0.32 \%$, respectively. However, no statistical differences were observed in the annual rate of change in BMD between the groups. During the follow-up period, the frequency of new osteoporosis was not significantly different between the GC group and the no-GC group (36 patients $(4.3 \%)$ vs. 6 patients $(6 \%) ; p=0.441)$. A comparison of the fractures occurring prior to the baseline BMD test and the incidental fractures occurring during the study is shown in Figure 2. There were 43 (5.2\%) new fractures in the GC group and $3(3 \%)$ new fractures in the no-GC group, with no significant difference between them. Among them, vertebral fractures were noted in 37 patients in the GC group and 2 patients in the no-GC group, accounting for $4.4 \%$ and $2 \%$ of the total patient counts, respectively.

Table 3. Annual change in BMD and new-onset osteoporosis rates between the GC users and the control group.

\begin{tabular}{|c|c|c|c|}
\hline Variable & GC $(N=833)$ & No-GC (N = 100) & $p$ Value \\
\hline \multicolumn{4}{|c|}{$\begin{array}{l}\text { Annual mean value of the absolute difference between baseline and } \\
\text { follow-up BMD }\left(\mathrm{g} / \mathrm{cm}^{2}\right)\end{array}$} \\
\hline Lumbar spine & $0.014 \pm 0.043$ & $0.01 \pm 0.042$ & 0.414 \\
\hline Femoral neck & $-0.003 \pm 0.028$ & $-0.005 \pm 0.02$ & 0.959 \\
\hline Total hip & $-0.003 \pm 0.031$ & $-0.003 \pm 0.015$ & 0.6 \\
\hline \multicolumn{4}{|l|}{ Annual $(\%)$ change in BMD $\left(\mathrm{g} / \mathrm{cm}^{2}\right)$} \\
\hline Lumbar spine & $1.76 \pm 4.9$ & $1.26 \pm 4.44$ & 0.393 \\
\hline Femoral neck & $-0.33 \pm 4.16$ & $-0.53 \pm 2.62$ & 0.6 \\
\hline Total hip & $-0.38 \pm 3.42$ & $-0.32 \pm 1.8$ & 0.453 \\
\hline New-onset osteoporosis, N. (\%) & $36(4.3)$ & $6(6)$ & 0.441 \\
\hline
\end{tabular}

BMD: bone densitometry, GC: glucocorticoid.

(a)

Prevalent osteoporotic fracture

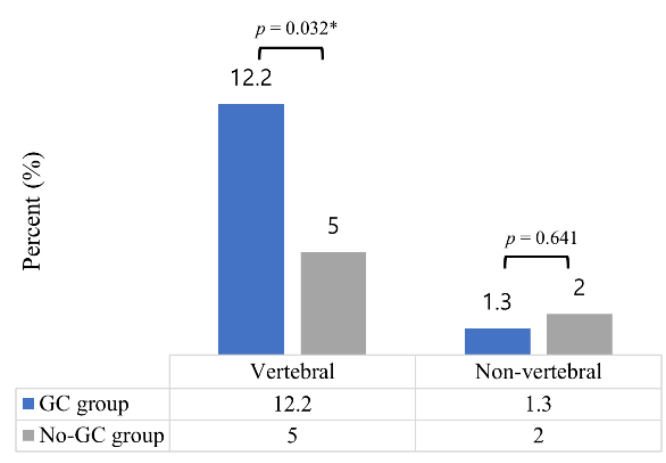

(b)

\section{Incident osteoporotic fracture}

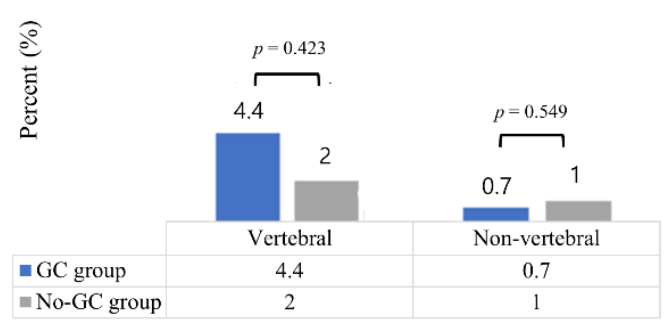

Figure 2. Comparison of vertebral and non-vertebral osteoporotic fractures. (a) Prevalent vertebral and non-vertebral fractures at baseline; (b) Incidental vertebral and non-vertebral fractures after baseline. GC: Glucocorticoid. ${ }^{*} p<0.05$.

\subsection{Factors Affecting Annual Changes in BMD}

Various factors influencing the annual change in the BMD of the lumbar spine, femoral neck, and total hip were analyzed using multiple linear regression analysis, as presented in Table 4. Factors known to affect changes in BMD (such as age, menopause, BMI, and smoking) and DAS28-ESR or DAS28-CRP differences between baseline and follow-up were 
included in the variables. The comparison of the DAS28-ESR at baseline and follow-up showed that there were significant differences between the two groups. In the GC group, the DAS28-ESR decreased at follow-up compared to that at baseline, whereas in the no-GC group, it increased marginally at follow-up $(-0.27 \pm 1$ vs. $0.83 \pm 0.71 ; p<0.001$, data not shown). The mean change in the DAS28-CRP level was not significantly different between the groups. Univariate linear regression analyses revealed that age, menopause, BMI, baseline DAS28-ESR, $\triangle$ DAS28-ESR, cumulative GC dose, and dietary vitamin D intake were significantly associated with annual changes in BMD. Multiple linear regression analyses using stepwise variable selection showed that baseline DAS28-ESR and $\triangle \mathrm{DAS28}$ ESR were related to annual changes in the BMD of the lumbar spine. In the femoral neck and total hip, only $\triangle \mathrm{DAS28}$-ESR was an influential variable affecting annual changes in BMD.

Table 4. Multiple linear regression analysis of risk factors associated with annualized BMD change.

\begin{tabular}{|c|c|c|c|c|c|c|}
\hline \multirow{2}{*}{ Variable } & \multicolumn{2}{|c|}{ Lumbar Spine } & \multicolumn{2}{|c|}{ Femoral Neck } & \multicolumn{2}{|c|}{ Total Hip } \\
\hline & $\beta$ & $p$ Value & $\beta$ & $p$ Value & $\beta$ & $p$ Value \\
\hline \multicolumn{7}{|l|}{ Univariate } \\
\hline Age & 0.041 & $0.013 *$ & -0.001 & 0.942 & 0 & 0.998 \\
\hline Menopause & 1.560 & $0.006^{*}$ & -0.387 & 0.421 & -0.003 & 0.994 \\
\hline BMI & -0.027 & 0.532 & -0.033 & 0.362 & -0.078 & $0.009 *$ \\
\hline Smoking & 1.01 & 0.136 & -0.188 & 0.734 & -0.088 & 0.846 \\
\hline Alcohol & 0.001 & 0.999 & -0.01 & 0.98 & 0.008 & 0.982 \\
\hline RF positivity & 0.085 & 0.801 & -0.001 & 0.998 & -0.03 & 0.897 \\
\hline Anti-CCP Ab positivity & 0.228 & 0.574 & 0.158 & 0.644 & 0.27 & 0.302 \\
\hline Disease duration & 0.001 & 0.543 & -0.001 & 0.583 & 0.001 & 0.474 \\
\hline Baseline DAS28-ESR & 0.59 & $0.002 *$ & 0.457 & $0.009 *$ & 0.218 & 0.099 \\
\hline Baseline DAS28-CRP & 0.379 & 0.109 & 0.192 & 0.369 & 0.048 & 0.768 \\
\hline$\triangle \mathrm{DAS} 28-\mathrm{ESR}$ & -1.9 & $<0.001 *$ & -0.936 & $<0.001 *$ & -1.02 & $<0.001 *$ \\
\hline$\triangle \mathrm{DAS} 28-\mathrm{CRP}$ & 0.379 & 0.109 & 0.192 & 0.369 & 0.048 & 0.768 \\
\hline Bone erosion & 0.538 & 0.103 & -0.008 & 0.769 & 0.129 & 0.565 \\
\hline Cumulative CS dose & 0.077 & $0.002 *$ & 0.029 & 0.163 & 0.113 & $0.002 *$ \\
\hline Calcium intake & -0.344 & 0.308 & 0.209 & 0.455 & 0.673 & 0.621 \\
\hline Vitamin D intake & 1.61 & $0.001 *$ & 0.964 & 0.016 & 0.052 & $0.04 *$ \\
\hline PPI use & 0.189 & 0.856 & 0.01 & 0.114 & 0.599 & 0.638 \\
\hline \multicolumn{7}{|l|}{ Multivariate } \\
\hline Age & 0.009 & 0.707 & 0.009 & 0.659 & 0.002 & 0.885 \\
\hline Menopause & -0.077 & 0.917 & -0.131 & 0.839 & -0.14 & 0.772 \\
\hline BMI & -0.031 & 0.585 & -0.047 & 0.367 & -0.018 & 0.637 \\
\hline Baseline DAS28-ESR & -0.593 & $0.006 *$ & -0.068 & 0.737 & 0.223 & 0.092 \\
\hline$\triangle \mathrm{DAS} 28-\mathrm{ESR}$ & -2.08 & $<0.001 *$ & -0.908 & $<0.001 *$ & -0.964 & $<0.001 *$ \\
\hline Cumulative GC dose & -0.003 & 0.914 & 0.051 & 0.072 & -0.003 & 0.906 \\
\hline Vitamin D intake & -0.119 & 0.842 & 0.619 & 0.26 & 0.309 & 0.445 \\
\hline
\end{tabular}

BMD: bone mineral density, BMI: body mass index, RF: rheumatoid factor, Anti-CCP Ab: anti-citrullinated protein antibody, ESR: erythrocyte sedimentation rate, CRP: C-reactive protein, DAS: disease activity score, GC: glucocorticoid, PPI: proton pump inhibitor, NA: not applicable. $\triangle$ DAS28-ESR is DAS28-ESR at the follow-up point minus baseline DAS28-ESR. $\triangle D A S 28-C R P$ is DAS28-CRP at the follow-up point minus baseline DAS28-CRP. ${ }^{*} p<0.05$.

\subsection{Annual BMD Changes Depending on the GC Dose}

Figure 3 shows the changes in BMD according to the mean daily GC dose in the GC group. Significant reductions in the BMD values of the femoral neck and total hip were observed in patients taking a dose of at least $2.5 \mathrm{mg}$ /day compared to those taking a dose of $<2.5 \mathrm{mg} /$ day. In the $\geq 2.5 \mathrm{mg}$ and $<2.5 \mathrm{mg}$ GC groups, the annual rates of change in the BMD of the femoral neck were $-0.84 \pm 3.63$ and $0.01 \pm 4.44$, respectively $(p=0.004)$, and those of the total hip were $-0.67 \pm 3.44$ and $-0.19 \pm 3.44$, respectively $(p=0.048)$. There was no difference in the annual rate of change in the BMD of the lumbar spine according to the GC dose. 


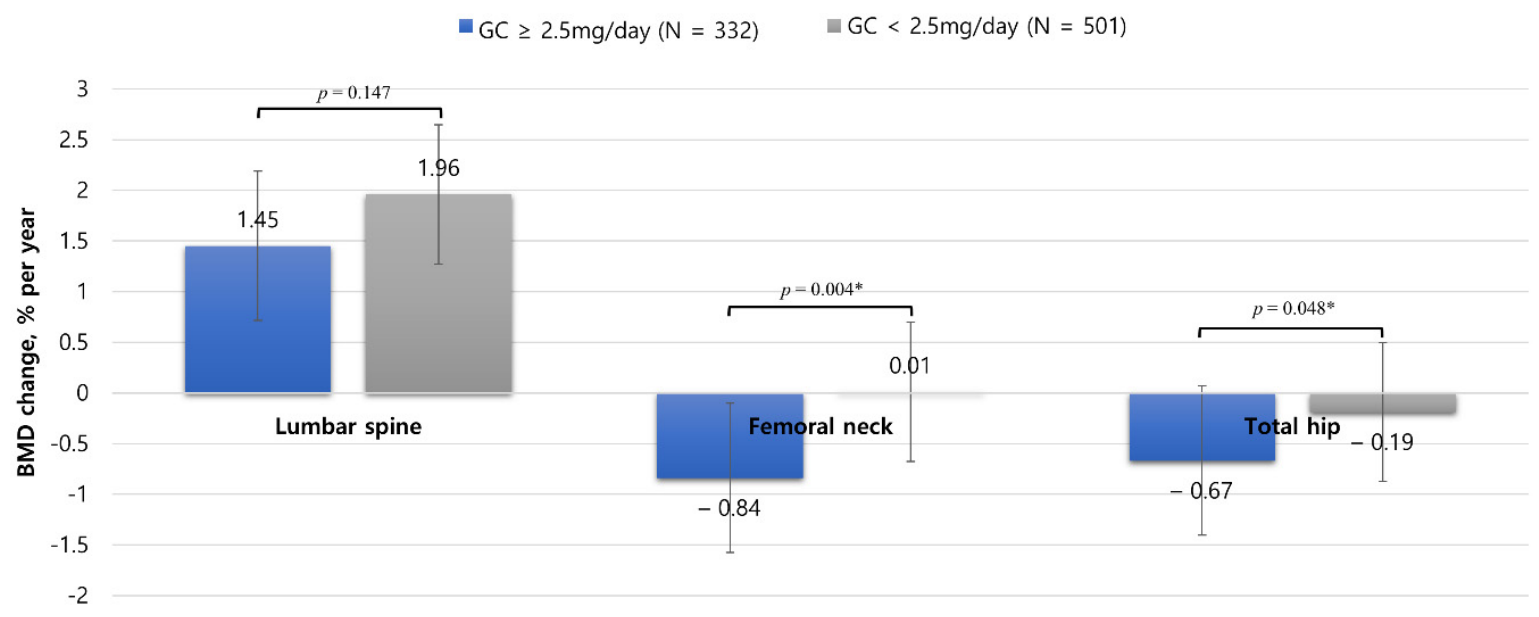

Figure 3. Annual rates of change in the bone mineral density of the lumbar spine, femoral neck, and total hip according to the mean daily glucocorticoid dose. Error bars represent the standard error of the mean. GC: Glucocorticoid, BMD: bone mineral density. ${ }^{*} p<0.05$.

\section{Discussion}

It is widely known that GCs can cause bone loss, reduce bone strength, and eventually lead to osteoporotic fractures; however, they exhibit protective roles due to their antiinflammatory actions against underlying diseases [13]. Therefore, the debate regarding the balance between the benefits and risks of GC therapy is ongoing, with no consensus regarding either opinion since their introduction in the 1950s. As we already know, GCs are widely used in chronic diseases because of their rapid anti-inflammatory effects [14-16]. Especially in RA, the treatment guidelines suggest the use of GCs for the shortest period possible as a bridging therapy until conventional DMARDs show efficacy ( $<3$ months) [17]. In actual clinical practice, the reduction or withdrawal of GCs is failing for various reasons; therefore, it is necessary to identify the deleterious effects of even low-dose GCs. This study analyzed the changes in BMD and the incidence of osteoporotic fractures among several adverse effects. To determine the effect of low-dose GCs on BMD, we compared patients undergoing low-dose GC therapy with those who discontinued GC therapy, rather than dividing them into never-/ever-exposed groups, because most patients receive GCs at the beginning of RA treatment. Although the no-GC group comprised past users rather than non-users, the risk from GCs would have had a minimal impact on the results of the study since the risk of osteoporosis and fractures decreases after cessation of GC therapy, and most participants were enrolled after a year of discontinuation of GCs [18].

The baseline disease activity, cumulative GC doses, and radiologic changes in the GC group increased compared to those in the no-GC group, which could be the reason for the inability to discontinue GC. The clinical difference between the two groups did not affect the BMD of the lumbar spine; however, there was a significant difference in the baseline BMD values of the femoral neck and total hip between the groups. Despite the high cumulative GC doses in the GC group, the result that the BMD of only some subregions decreased was inconsistent with that reported in a previous study [19]. In a previous meta-analysis, there was no disagreement on the conclusion that the BMD of all areas decreased with GC therapy; however, recently, other factors such as anti-bone-resorption agents and disease activity have been reported to outperform the bone-reduction effect of GCs [20,21]. Several studies on patients with RA treated with GCs have shown that the use of anti-bone-resorption agents increases the BMD and markers of bone turnover of the lumbar spine [22,23]. Another study showed that the markers of bone metabolism have different influencing abilities depending on the skeletal sites; thus, BMD does not decrease uniformly in all areas [24]. Although the effect on cumulative GC doses cannot be excluded, it may be necessary to closely analyze the relationship between the pathophysiology of RA 
and bone metabolism, as there have been studies in the literature to indicate that patients with RA have lesser changes in lumbar BMD compared to other subregions [25].

In both groups, the annual rate of change in the BMD of the lumbar spine increased, while that of the femoral neck and total hip decreased. In many older adults, degenerative change in the lumbar spine increases its BMD falsely, rather than causing an actual increase [26]. Notably, unlike in previous studies, low-dose GCs did not significantly affect the annual rate of change in BMD in our study. There was no increase in the incidence of new-onset osteoporosis or osteoporotic fractures during the follow-up period in the GC group. This is in contrast to the hypothesis that the use of GCs in patients with RA increases the risk of osteoporosis and osteoporotic fractures [27,28]. Similarly to our study, other studies have advocated the use of low-dose GCs in patients with RA based on bone loss [29-33]. This emphasizes that our findings are reliable, because previous studies included patients with early RA or had small samples or a short follow-up period [34]. Considering this, the long-term follow-up observation in this study is a significant advantage.

From the above results alone, it cannot be concluded that low-dose GCs have no effect on BMD reduction. Given the differences in the baseline characteristics between the two groups, factors other than GCs may have affected the annual rate of change in BMD. Several independent factors associated with the decreasing annual rate of change in BMD were identified using univariate statistical analysis. The analysis showed that $\triangle$ DAS28-ESR and cumulative GC dose were associated with the annual rates of change in the BMD of the lumbar spine, femoral neck, and total hip. In addition, age, menopausal status, BMI, baseline DAS28-ESR, and use of vitamin D supplements were found to be risk factors for some of the subregions. These findings are similar to those of previous studies [35-39]. In the multivariate approach calibrating the interrelated variables, only baseline DAS28-ESR and $\triangle$ DAS28-ESR contributed to BMD reduction. Thus, among the potential factors, achieving remission status by controlling the disease activity is the most critical factor in preventing bone loss. This result is consistent with the findings that tighter control of disease activity is the greatest contributor in improving the outcome of RA with the discovery of various biological agents for the treatment of RA over the past 30 years [40]. Data from the past decade have shown a potential positive effect on BMD in patients with RA whose disease activity is well-controlled by the use of biological agents, regardless of GC therapy [41]. In a recent study, RA patients with persistent remission status displayed similar changes in BMD even when compared with normal controls $[42,43]$. Furthermore, several studies conducted on other chronic inflammatory diseases, such as systemic lupus erythematosus and asthma, have also shown that proper management of the disease is clinically important to preventing BMD reduction, rather than discontinuing the use of GCs [44,45].

Since there were too many factors affecting the disease activity of RA and bone metabolism, we performed further analysis to minimize bias in the interpretation of the results. To compensate for the differences in baseline characteristics of the two groups, we additionally conducted propensity score matching (PSM) with factors that directly affect osteoporosis and fracture risk, including age, sex, seropositivity, and DMARDs. Comparison by PSM also showed no differences in the annual rate of change in BMD between the GC group and the no-GC group. Furthermore, the result that $\triangle \mathrm{DAS} 28$-ESR was an independent factor influencing the annual rate of change in the BMD remained unchanged after PSM (Tables S1-S3). Despite the effects of confounding factors, the results of this study were similar to those after PSM. Collectively, various factors affecting bone metabolism in patients with RA did not ultimately change the effect on GC-induced BMD reduction and fracture risk.

As mentioned above, it is essential to maintain a well-controlled status in chronic diseases, which requires continuous management after diagnosis; however, this does not mean that the use of GCs is unconditionally justified. In addition to the incidence of osteoporosis and osteoporotic fractures, GCs are associated with the occurrence of other complications such as hyperglycemia, hypertension, glaucoma, and mood changes; 
therefore, personalized treatment strategies should be developed. Just as the effectiveness of RA treatments has been dramatically improved by introducing paradigm shifts in applying treat-to-target strategies as a personalized approach, introducing a personalized approach can lead to various benefits in the use of GCs as well [46]. Thus, our study further analyzed whether there was a dose-dependent change in BMD. In line with recommended guidelines to prevent further fractures in all patients with previous osteoporosis fractures taking glucocorticoids, a very-low-GC group was classified based on $2.5 \mathrm{mg}$ prednisone or an equivalent [47]. We found that even among patients consuming doses of $\leq 7.5 \mathrm{mg}$, which is generally considered a low dose, there was a significantly lesser decrease in the annual rate of change in BMD in the $<2.5 \mathrm{mg}$ GC group than in the $\geq 2.5 \mathrm{mg}$ GC group. This means that a very low dose of GC $(2.5 \mathrm{mg})$ does not significantly decrease BMD, but it has a favorable effect on maintaining the remission status of RA. A previous study reported that the incidence of vertebral and non-vertebral fractures in patients with RA may be higher, even in those treated with an oral GC dose of $2.5 \mathrm{mg}$, than that in healthy controls, and this analysis was criticized as having a limitation of not considering the disease activity of RA [18]. In addition, a study conducted on RA patients who achieved clinical remission with the first biologic DMARD reported that the group who stopped GC had a longer survival time on biologic DMARD [48]. However, since this study included relatively young patients using biologic DMARD, these results cannot be broadly interpreted as indicating a requirement for $\mathrm{GC}$ to be discontinued to reach clinical remission in all RA patients.

Our study is novel because it is the first study to report that very-low-dose GCs $(<2.5 \mathrm{mg} /$ day) are relatively safe in terms of controlling the disease activity and preventing osteoporosis and fractures. It is best to maintain clinical remission while reducing the use of GCs as much as possible through various combinations of medications; however, there is no need to discontinue GCs forcefully due to concerns of its adverse effects if the inflammation is not fully controlled. Furthermore, maintaining very-low-dose GC therapy requires meeting other conditions to prevent $\mathrm{BMD}$ reduction, such as calcium and vitamin D supplementation and appropriate pharmacological treatment of osteoporosis [49]. Finally, this study concludes that the benefits of GC therapy in attenuating inflammation compensate for the risk of osteoporosis when sufficient preventive measures are taken to prevent bone loss in patients with RA.

The limitations of our study include its retrospective single-center design. Due to these limitations, there were differences in the baseline characteristics and some DMARDs of the two groups. Factors affecting osteoporosis and fracture risk due to direct effects on bones, such as anti-TNF agents, bisphosphonate, and autoantibody positivity, were also different. Autoantibody positivity is known to be a negative prognostic factor for bone erosions and osteoporosis, as well as RA; therefore, patients with seropositive RA would have needed to maintain GC to achieve low disease activity or remission status. To overcome this limitation, we additionally conducted propensity score matching (PSM) for age, sex, seropositivity, and some DMARDs, which showed no differences in the annual rate of change in BMD between the GC group and the no-GC group. Nevertheless, the inability to match all demographic and clinical characteristics of patients with RA enrolled in this study remained a limitation. Another limitation is that the study did not include all patients with RA taking GCs, but only those who underwent BMD testing. Therefore, the proportion of postmenopausal women increased compared to the known prevalence of RA by sex and age, which inevitably served as a selection bias. Moreover, osteoporosispreventive treatment was not administrated equally to all patients, so the dosage of vitamin $\mathrm{D}$ or calcium varied among patients. In addition, functional disability, which affects the occurrence of fractures, was not evaluated. This study covered a vast period of 20 years, during which there have been many advances in medications; thus, the frequency of use of the most recent medications, such as teriparatide and denosumab for osteoporosis treatment, and JAK inhibitors for RA treatment, was relatively low. 


\section{Conclusions}

Our study suggest that the net effect of low-dose GCs compensates for its detrimental effects on BMD in patients with RA. Tight control of disease activity, even with GC therapy, may reduce the long-term effects of bone inflammation and halt progressive bone loss. In conclusion, it is recommended that rheumatologists optimize the use of GCs to minimize the associated adverse effects, including osteoporosis and osteoporotic fractures, while maintaining clinical remission or improving anti-inflammatory efficacy through GC therapy.

Supplementary Materials: The following are available online at https:/ /www.mdpi.com/article/ $10.3390 / \mathrm{jcm} 10132944 / \mathrm{s} 1$, Table S1: Baseline demographic and clinical characteristics of patients with RA after propensity score matching, Table S2. Annual change in BMD and new-onset osteoporosis rates between the GC users and the control group after propensity score matching; Table S3. Multiple linear regression analysis of risk factors associated with annualized BMD change after propensity score matching.

Author Contributions: Conceptualization, J.-W.K. and C.-H.S.; formal analysis, J.-W.K., J.-Y.J., H.-A.K. and C.-H.S.; investigation, J.-W.K., J.-Y.J., H.-A.K. and C.-H.S.; writing-original draft preparation, J.-W.K.; writing - review and editing, J.-W.K. and C.-H.S.; funding acquisition, C.-H.S. All authors have read and agreed to the published version of the manuscript.

Funding: This research was supported by a grant from the Korea Health Technology R\&D Project through the Korea Health Industry Development Institute, funded by the Ministry of Health and Welfare, Republic of Korea (grant number: HI16C0992).

Institutional Review Board Statement: This study was approved by the Institutional Review Board of Ajou University Hospital (AJIRB-MED-MDB-21-109).

Informed Consent Statement: Considering the retrospective nature of the study, the Ethics Committee agreed to waive the consent of individual patients.

Data Availability Statement: The datasets generated for this study are not available due to the data protection law.

Conflicts of Interest: The authors declare no conflict of interest.

\section{References}

1. Firestein, G.S. Evolving concepts of rheumatoid arthritis. Nat. Cell Biol. 2003, 423, 356-361. [CrossRef]

2. Falconer, J.; Murphy, A.N.; Young, S.P.; Clark, A.R.; Tiziani, S.; Guma, M.; Buckley, C.D. Review: Synovial Cell Metabolism and Chronic Inflammation in Rheumatoid Arthritis. Arthritis Rheumatol. 2018, 70, 984-999. [CrossRef]

3. Shim, J.-H.; Stavre, Z.; Gravallese, E.M. Bone loss in rheumatoid arthritis: Basic mechanisms and clinical implications. Calcif Tissue Int. 2018, 102, 533-546. [CrossRef]

4. Raterman, H.G.; Lems, W.F. Pharmacological Management of Osteoporosis in Rheumatoid Arthritis Patients: A Review of the Literature and Practical Guide. Drugs Aging 2019, 36, 1061-1072. [CrossRef]

5. Strehl, C.; Van Der Goes, M.C.; Bijlsma, J.W.; Jacobs, J.W.G.; Buttgereit, F. Glucocorticoid-targeted therapies for the treatment of rheumatoid arthritis. Expert Opin. Investig. Drugs 2017, 26, 187-195. [CrossRef] [PubMed]

6. Manolagas, S.C.; Weinstein, R.S. New Developments in the Pathogenesis and Treatment of Steroid-Induced Osteoporosis. J. Bone Miner. Res. 1999, 14, 1061-1066. [CrossRef]

7. Wang, Y.; Zhao, R.; Gu, Z.; Dong, C.; Guo, G.; Li, L. Effects of glucocorticoids on osteoporosis in rheumatoid arthritis: A systematic review and meta-analysis. Osteoporos. Int. 2020, 31, 1401-1409. [CrossRef]

8. Lindner, L.; Callhoff, J.; Alten, R.; Krause, A.; Ochs, W.; Zink, A.; Albrecht, K. Osteoporosis in patients with rheumatoid arthritis: Trends in the German National Database 2007-2017. Rheumatol. Int. 2020, 40, 2005-2012. [CrossRef] [PubMed]

9. Van Everdingen, A.A.; Siewertsz Van Reesema, D.R.; Jacobs, J.W.; Bijlsma, J.W.J. Low-dose glucocorticoids in early rheumatoid arthritis: Discordant effects on bone mineral density and fractures? Clin. Exp. Rheumatol. 2003, 21, 155-160. [PubMed]

10. Cheng, T.-T.; Lai, H.-M.; Yu, S.-F.; Chiu, W.-C.; Hsu, C.-Y.; Chen, J.-F.; Chen, Y.-C. The impact of low-dose glucocorticoids on disease activity, bone mineral density, fragility fractures, and 10-year probability of fractures in patients with rheumatoid arthritis. J. Investig. Med. 2018, 66, 1004-1007. [CrossRef] [PubMed]

11. Buttgereit, F.; Silva, J.A.P.D.; Boers, M.; Burmester, G.-R.; Cutolo, M.; Jacobs, J.; Kirwan, J.; Köhler, L.; Van Riel, P.; Vischer, T.; et al. Standardised nomenclature for glucocorticoid dosages and glucocorticoid treatment regimens: Current questions and tentative answers in rheumatology. Ann. Rheum. Dis. 2002, 61, 718-722. [CrossRef] 
12. World Health Organization. Assessment of fracture risk and its application to screening for postmenopausal osteoporosis: Report of a WHO study group. World Health Organ. Tech. Rep. Ser. 1994, 843, 1-129.

13. Lane, N.E. Glucocorticoid-Induced Osteoporosis: New Insights into the Pathophysiology and Treatments. Curr. Osteoporos. Rep. 2019, 17, 1-7. [CrossRef] [PubMed]

14. Hartman, L.; Rasch, L.A.; Klausch, T.; Bijlsma, H.W.J.; Christensen, R.; Smulders, Y.M.; Ralston, S.H.; Buttgereit, F.; Cutolo, M.; Da Silva, J.A.P.; et al. Harm, benefit and costs associated with low-dose glucocorticoids added to the treatment strategies for rheumatoid arthritis in elderly patients (GLORIA trial): Study protocol for a randomised controlled trial. Trials $2018,19,67$. [CrossRef]

15. Lodder, M.C.; De Jong, Z.; Kostense, P.J.; Molenaar, E.T.H.; Staal, K.; E Voskuyl, A.; Hazes, J.M.W.; Dijkmans, B.A.C.; Lems, W.F. Bone mineral density in patients with rheumatoid arthritis: Relation between disease severity and low bone mineral density. Ann. Rheum. Dis. 2004, 63, 1576-1580. [CrossRef] [PubMed]

16. Gough, A.K.; Emery, P.; Holder, R.; Lilley, J.; Eyre, S. Generalised bone loss in patients with early rheumatoid arthritis. Lancet 1994, 344, 23-27. [CrossRef]

17. Smolen, J.S.; Landewé, R.B.M.; Bijlsma, J.W.J.; Burmester, G.R.; Dougados, M.; Kerschbaumer, A.; McInnes, I.B.; Sepriano, A.; van Vollenhoven, R.F.; de Wit, M.; et al. EULAR recommendations for the management of rheumatoid arthritis with synthetic and biological disease-modifying antirheumatic drugs: 2019 update. Ann. Rheum. Dis. 2020, 79, 685-699. [CrossRef]

18. Van Staa, T.; Leufkens, H.; Abenhaim, L.; Zhang, B.; Cooper, C. Use of oral corticosteroids and risk of fractures. J. Bone Miner. Res. 2000, 15, 993-1000. [CrossRef] [PubMed]

19. Van Staa, T.P.; Leufkens, H.; Cooper, C. The epidemiology of corticosteroid-induced osteoporosis: A meta-analysis. Osteoporos Int. 2002, 13, 777-787. [CrossRef]

20. Lee, Y.H.; Woo, J.-H.; Choi, S.J.; Ji, J.D.; Song, G.G. Effects of low-dose corticosteroids on the bone mineral density of patients with rheumatoid arthritis: A meta-analysis. J. Investig. Med. 2008, 56, 1011-1018. [CrossRef] [PubMed]

21. Blavnsfeldt, A.-B.G.; de Thurah, A.; Thomsen, M.D.; Tarp, S.; Langdahl, B.; Hauge, E.-M. The effect of glucocorticoids on bone mineral density in patients with rheumatoid arthritis: A systematic review and meta-analysis of randomized, controlled trials. Bone 2018, 114, 172-180. [CrossRef]

22. Van der Goes, M.C.; Jacobs, J.W.; Jurgens, M.S.; Bakker, M.F.; van der Veen, M.J.; van der Werf, J.H.; Welsing, P.M.J.; Bijlsma, J.W.J. Are changes in bone mineral density different between groups of early rheumatoid arthritis patients treated according to a tight control strategy with or without prednisone if osteoporosis prophylaxis is applied? Osteoporos. Int. 2013, 24, 1429-1436. [CrossRef] [PubMed]

23. A Capell, H.; Madhok, R.; A Hunter, J.; Porter, D.; Morrison, E.; Larkin, J.; A Thomson, E.; Hampson, R.; Poon, F.W. Lack of radiological and clinical benefit over two years of low dose prednisolone for rheumatoid arthritis: Results of a randomised controlled trial. Ann. Rheum. Dis. 2004, 63, 797-803. [CrossRef]

24. Tomizawa, T.; Ito, H.; Murata, K.; Hashimoto, M.; Tanaka, M.; Murakami, K.; Nishitani, K.; Azukizawa, M.; Okahata, A.; Doi, K.; et al. Distinct biomarkers for different bones in osteoporosis with rheumatoid arthritis. Arthritis Res. 2019, 21, 1-9. [CrossRef]

25. Shibuya, K.; Hagino, H.; Morio, Y.; Teshima, R. Cross-Sectional and Longitudinal Study of Osteoporosis in Patients with Rheumatoid Arthritis. Clin. Rheumatol. 2002, 21, 150-158. [CrossRef]

26. Liu, G.; Peacock, M.; Eilam, O.; Dorulla, G.; Braunstein, E.; Johnston, C.C. Effect of osteoarthritis in the lumbar spine and hip on bone mineral density and diagnosis of osteoporosis in elderly men and women. Osteoporos Int. 1997, 7, 564-569. [CrossRef]

27. Shen, G.; Ren, H.; Qiu, T.; Liang, D.; Wei, Q.; Tang, J.; Zhang, Z.; Yao, Z.; Zhao, W.; Jiang, X. Effect of glucocorticoid withdrawal on glucocorticoid inducing bone impairment. Biochem. Biophys. Res. Commun. 2016, 477, 1059-1064. [CrossRef] [PubMed]

28. Aeberli, D.; Fankhauser, N.; Zebaze, R.; Bonel, H.; Möller, B.; Villiger, P. Effect of rheumatoid arthritis and age on metacarpal bone shaft geometry and density: A longitudinal pQCT study in postmenopausal women. Semin. Arthritis Rheum. 2020, 50, 220-227. [CrossRef]

29. Jacobs, J.W.; Bijlsma, J.W.; Van Laar, J.M. Glucocorticoids in Early Rheumatoid Arthritis: Are the Benefits of Joint-Sparing Effects Offset by the Adverse Effect of Osteoporosis? The Effects on Bone in the Utrecht Study and the CAMERA-II Study. Neuroimmunomodulation 2015, 22, 66-71. [CrossRef] [PubMed]

30. Hall, G.M.; Spector, T.D.; Griffin, A.J.; Jawad, A.S.M.; Hall, M.L.; Doyle, D.V. The effect of rheumatoid arthritis and steroid therapy on bone density in postmenopausal women. Arthritis Rheum. 1993, 36, 1510-1516. [CrossRef]

31. Buckley, L.M.; Leib, E.S.; Cartularo, K.S.; Vacek, P.M.; Cooper, S.M. Effects of low dose corticosteroids on the bone mineral density of patients with rheumatoid arthritis. J. Rheumatol. 1995, 22, 1055-1059.

32. Balasubramanian, A.; Wade, S.; Adler, R.; Lin, C.J.F.; Maricic, M.; O’Malley, C.D.; Saag, K.; Curtis, J.R. Glucocorticoid exposure and fracture risk in patients with new-onset rheu-matoid arthritis. Osteoporos. Int. 2016, 27, 3239-3249. [CrossRef]

33. Book, C.; Karlsson, M.; Åkesson, K.; Jacobsson, L. Disease activity and disability but probably not glucocorticoid treatment predicts loss in bone mineral density in women with early rheumatoid arthritis. Scand. J. Rheumatol. 2008, 37, 248-254. [CrossRef] [PubMed]

34. Guler-Yuksel, M.; Bijsterbosch, J.; Goekoop-Ruiterman, Y.P.M.; De Vries-Bouwstra, J.K.; Hulsmans, H.M.J.; De Beus, W.M.; Han, K.H.; Breedveld, F.C.; Dijkmans, B.A.C.; Allaart, C.F.; et al. Changes in bone mineral density in patients with recent onset, active rheumatoid arthritis. Ann. Rheum. Dis. 2008, 67, 823-828. [CrossRef] [PubMed] 
35. Tawaratsumida, H.; Setoguchi, T.; Arishima, Y.; Ohtsubo, H.; Akimoto, M.; Ishidou, Y.; Nagano, S.; Taketomi, E.; Sunahara, N.; Komiya, S. Risk factors for bone loss in patients with rheumatoid arthritis treated with biologic disease-modifying anti-rheumatic drugs. BMC Res. Notes 2017, 10, 765. [CrossRef]

36. Başkan, B.M.; Sivas, F.; Alemdaroğlu, E.; Duran, S.; Ozoran, K. Association of bone mineral density and vertebral deformity in patients with rheumatoid arthritis. Rheumatol. Int. 2007, 27, 579-584. [CrossRef]

37. Chen, J.; Liu, W.; Lin, Q.; Chen, L.; Yin, J.; Huang, H. Vitamin D deficiency and low bone mineral density in native C hinese rheumatoid arthritis patients. Int. J. Rheum. Dis. 2014, 17, 66-70. [CrossRef]

38. Okano, T.; Inui, K.; Tada, M.; Sugioka, Y.; Mamoto, K.; Wakitani, S.; Koike, T.; Nakamura, H. Loss of lean body mass affects low bone mineral density in patients with rheumatoid ar-thritis-results from the TOMORROW study. Mod. Rheumatol. 2017, 27, 946-952. [CrossRef] [PubMed]

39. Zhang, X.; Dai, Z.; Lau, E.H.Y.; Cui, C.; Lin, H.; Qi, J.; Ni, W.; Zhao, L.; Lv, Q.; Gu, J.; et al. Prevalence of bone mineral density loss and potential risk factors for osteopenia and oste-oporosis in rheumatic patients in China: Logistic regression and random forest analysis. Ann. Transl. Med. 2020, 8, 226. [CrossRef]

40. Mease, P.J. Improving the Routine Management of Rheumatoid Arthritis: The Value of Tight Control. J. Rheumatol. 2010, 37, 1570-1578. [CrossRef]

41. Theander, L.; Willim, M.; Nilsson, J. Åke; Karlsson, M.; E Åkesson, K.; Jacobsson, L.T.H.; Turesson, C. Changes in bone mineral density over 10 years in patients with early rheumatoid arthritis. RMD Open 2020, 6, e001142. [CrossRef]

42. Yoshii, I.; Chijiwa, T.; Sawada, N. Rheumatoid arthritis in tight disease control is no longer risk of bone mineral density loss. Osteoporos. Sarcopenia 2020, 6, 75-81. [CrossRef]

43. Hafez, E.A.; Mansour, H.E.; Hamza, S.H.; Moftah, S.G.; Younes, T.B.; Ismail, M.A. Bone Mineral Density Changes in Patients with Recent-Onset Rheumatoid Arthritis. Clin. Med. Insights Arthritis Musculoskelet. Disord. 2011, 4, 87-94. [CrossRef]

44. Lee, C.; Almagor, O.; Dunlop, D.D.; Manzi, S.; Spies, S.; Chadha, A.B.; Ramsey-Goldman, R. Disease damage and low bone mineral density: An analysis of women with systemic lupus erythematosus ever and never receiving corticosteroids. Rheumatology 2005, 45, 53-60. [CrossRef] [PubMed]

45. Chen, W.; Johnson, K.M.; Fitzgerald, J.M.; Sadatsafavi, M.; Leslie, W.D. Long-term effects of inhaled corticosteroids on bone mineral density in older women with asthma or COPD: A registry-based cohort study. Arch. Osteoporos. 2018, 13, 116. [CrossRef]

46. Batko, B.; Batko, K.; Krzanowski, M.; Żuber, Z. Physician adherence to treat-to-target and practice guidelines in rheumatoid arthritis. J. Clin. Med. 2019, 8, 1416. [CrossRef] [PubMed]

47. Buckley, L.; Guyatt, G.; Fink, H.A.; Cannon, M.; Grossman, J.; Hansen, K.E.; Humphrey, M.B.; Lane, N.E.; Magrey, M.; Miller, M.; et al. 2017 American college of rheumatology guideline for the prevention and treatment of glucocorticoid-induced osteoporosis. Arthritis Rheumatol. 2017, 69, 1521-1537. [CrossRef] [PubMed]

48. Fornaro, M.; Cacciapaglia, F.; Lopalco, G.; Venerito, V.; Iannone, F. Predictors of long-term clinical remission in rheumatoid arthritis. Eur. J. Clin. Investig. 2021, 51. [CrossRef]

49. Weaver, C.M.; Alexander, D.D.; Boushey, C.J.; Dawson-Hughes, B.; Lappe, J.M.; LeBoff, M.S.; Liu, S.; Looker, A.C.; Wallace, T.C.; Wang, D.D. Calcium plus vitamin D supplementation and risk of fractures: An updated meta-analysis from the National Osteoporosis Foundation. Osteoporos. Int. 2016, 27, 367-376. [CrossRef] 